\title{
EXACT FINITE-DIMENSIONAL FILTERS FOR MAXIMUM LIKELIHOOD PARAMETER ESTIMATION OF CONTINUOUS-TIME LINEAR GAUSSIAN SYSTEMS*
}

\author{
ROBERT J. ELLIOTT ${ }^{\dagger}$ AND VIKRAM KRISHNAMURTHY $\ddagger$
}

\begin{abstract}
In this paper, we derive a new class of finite-dimensional filters for integrals and stochastic integrals of moments of the state for continuous-time linear Gaussian systems. Apart from being of significant mathematical interest, these new filters can be used with the expectation maximization (EM) algorithm to yield maximum likelihood estimates of the model parameters.
\end{abstract}

Key words. stochastic systems, finite-dimensional filters, Kalman filter, expectation maximization algorithm, parameter estimation

AMS subject classifications. 93E11, 93E12, 93E10, 60G35

PII. S036301299529255X

1. Introduction. The Kalman filter is widely used in engineering, economics, and other fields. It considers linear Gaussian dynamics for the state $x$ and observation $y$ processes of the form

$$
\begin{aligned}
& d x_{t}=A_{t} x_{t} d t+B_{t} d w_{t}, \quad x_{0} \in \mathbb{R}^{m} \\
& d y_{t}=C_{t} x_{t} d t+D_{t} d v_{t}, \quad y_{0}=0 \in \mathbb{R}^{n} .
\end{aligned}
$$

The Kalman filter determines the conditional density of the unobserved signal $x_{t}$ given the observations to time $t, \mathcal{Y}_{t}=\sigma\left\{y_{s}: s \leq t\right\}$, as a Gaussian random variable with mean $m_{t}$ and variance $\Sigma_{t}$. Here $m_{t}$ is the conditional mean of $x_{t}$ given $\mathcal{Y}_{t}$, and $\Sigma_{t}$ is the conditional variance of $x_{t}$, although $\Sigma_{t}$ turns out to be deterministic and given by the Riccati equation.

To apply the Kalman filter, however, the parameters of the model, that is, the entries in the matrices $A, B, C$, and $D$, need to be known. Maximum likelihood estimation of these parameters via the expectation maximization (EM) algorithm has been studied in discrete time in [1], [2], [3] and in continuous time in [9]. In continuous time, the EM algorithm requires computation of the filtered estimates of quantities such as $\int_{0}^{t} x_{s} d x_{s}^{0}, \int_{0}^{t} x_{s} d y_{s}^{0}, \int_{0}^{t} x_{s} x_{s}^{0} d s$.

In all the existing literature on parameter estimation of linear Gaussian models via the EM algorithm, filtered estimates of the above quantities are computed via Kalman smoothing, which requires large memory in any numerical implementation. The main result of this paper, Theorem 3.10, provides finite-dimensional filters for (the components of) such integral processes. In fact, as pointed out in section 4, finitedimensional filters exist for integrals and stochastic integrals of moments of all orders

* Received by the editors September 29, 1995; accepted for publication (in revised form) August 2, 1996.

http://www.siam.org/journals/sicon/35-6/29255.html

$\dagger$ Department of Mathematical Sciences, University of Alberta, Edmonton, T6G 2G1, AB, Canada (relliott@gpu.srv.ualberta.ca). The research of this author was supported in part by NSERC grant A7964, the University of Melbourne, and the Co-operative Research Center for Sensor Signal and Information Processing.

${ }^{\ddagger}$ Department of Electrical and Electronic Engineering, University of Melbourne, Parkville, Victoria 3052, Australia (vikram@mullian.ee.mu.oz.au). The research of this author was supported by an ARC grant, ATERB, and the Co-operative Research Center for Sensor Signal and Information Processing. 
of the state process. In [18] and [19], finite-dimensional filters are described using Lie algebra methods, for time integrals of powers of the state variable. However, our techniques are quite different; we also obtain results for stochastic integrals of powers of the process, and we apply our results to maximum likelihood parameter estimation. Few finite-dimensional filters are known, so our results were certainly a surprise.

The analogous results in discrete time are derived in a companion paper [17]. The continuous-time methods and results in this paper are different from, and not just adaptations of, the discrete-time results in [17]. As described in [17], estimation of the state-space parameters arises in several areas of signal processing and control, including multisensor signal enhancement and speech coding, and also in econometrics.

Parameter estimation for diffusion processes is nicely discussed in [9]; this work also includes a discussion of the EM algorithm. Section 5 describes this application of the results of section 4. The techniques of this paper extend those for Markov chains presented in [5] and the recent book, [6].

The paper is organized as follows: in section 2 we present a standard measure change that simplifies the derivation of our filters. In section 3 the new finitedimensional filters are derived. Section 4 derives a finite-dimensional filter for higher order moments of the state. In section 5, the filters in section 3 are used to implement a filter-based EM algorithm for maximum likelihood parameter estimation.

2. Dynamics. Consider the classical linear Gaussian model for the signal model and observation processes. That is, the signal $\left\{x_{t}\right\}, t \geq 0$, is described by the equation

$$
d x_{t}=A_{t} x_{t} d t+B_{t} d w_{t}, \quad x_{0} \in \mathbb{R}^{m},
$$

and the observation process $\left\{y_{t}\right\}, t \geq 0$, is described as

$$
d y_{t}=C_{t} x_{t} d t+D_{t} d v_{t}, \quad y_{0}=0 \in \mathbb{R}^{n} .
$$

Here $w$ and $v$ are independent $r$-dimensional and $n$-dimensional Brownian motions, respectively, defined on a probability space $(\Omega, \mathcal{F}, P)$. Further, $w$ and $v$ are independent of $x_{0}$. We assume that $x_{0}$ is a random variable with normal density $\pi_{0}(x)$, which is $N\left(\hat{x}_{0}, P_{0}\right)$.

The matrix functions $A_{t} \in \mathbb{R}^{m \times m}, B_{t} \in \mathbb{R}^{m \times r}, C_{t} \in \mathbb{R}^{n \times m}$, and $D_{t} \in \mathbb{R}^{n \times n}$ are measurable functions of $t$. We assume $D_{t}$ is a positive definite matrix.

We model the above dynamics by supposing that initially we have a probability space $(\Omega, \mathcal{F}, \bar{P})$ such that under $\bar{P}$

1. $w$ is $r$-dimensional Brownian motion and $\left\{x_{t}\right\}$ is defined by (2.1).

2. $\left\{y_{t}\right\}$ is $n$-dimensional Brownian motion, independent of $w$ and $x_{0}$, and having quadratic variation $\langle y\rangle_{t}=D_{t}>0$; i.e., $D_{t}$ is a positive definite matrix.

Notation. Consider the complete right continuous filtrations

$$
\begin{aligned}
\mathcal{G}_{t} & =\sigma\left\{x_{s}, y_{s}: s \leq t\right\}, \\
\mathcal{Y}_{t} & =\sigma\left\{y_{s}: s \leq t\right\}
\end{aligned}
$$

Vectors $x, y$ will be considered as column vectors. Write

$$
\nabla=\left(\frac{\partial}{\partial x_{1}}, \frac{\partial}{\partial x_{2}}, \ldots, \frac{\partial}{\partial x_{n}}\right)^{0}
$$


For any function $g: \mathbb{R}^{m} \rightarrow \mathbb{R}$, write

$$
\nabla^{2} g=\left[\begin{array}{ccc}
\frac{\partial^{2} g}{\partial x_{1}^{2}} & \cdots & \frac{\partial^{2} g}{\partial x_{1} \partial x_{m}} \\
\vdots & \vdots & \vdots \\
\frac{\partial^{2} g}{\partial x_{m} \partial x_{1}} & \cdots & \frac{\partial^{2} g}{\partial x_{m}^{2}}
\end{array}\right]
$$

For a vector field $g(x)=\left[g_{1}(x) g_{2}(x) \cdots g_{m}(x)\right]^{0}$ defined on $\mathbb{R}^{m}$, define

$$
\operatorname{div}(g)=\frac{\partial g_{1}}{\partial x_{1}}+\frac{\partial g_{2}}{\partial x_{2}}+\cdots+\frac{\partial g_{m}}{\partial x_{m}} .
$$

Consider the exponential

$$
\Lambda_{t}=\exp \left(\int_{0}^{t}\left(C_{s} x_{s}\right)^{0}\left(D_{s}^{-1}\right)^{0} D_{s}^{-1} d y_{s}-\frac{1}{2} \int_{0}^{t} x_{s}^{0} C_{s}^{0}\left(D_{s}^{-1}\right)^{0} D_{s}^{-1} C_{s} x_{s} d s\right) .
$$

Then

$$
d \Lambda_{t}=\Lambda_{t} x_{t}^{0} C_{t}^{0}\left(D_{s}^{-1}\right)^{0} D_{s}^{-1} d y_{t}
$$

and $\overline{\mathbf{E}}\left\{\Lambda_{t}\right\}=1$, where $\overline{\mathbf{E}}$ denotes expectation under $\bar{P}$.

If we define a measure $P$ in terms of $\bar{P}$ by setting

$$
\left.\frac{d P}{d \bar{P}}\right|_{\mathrm{G}_{t}}=\Lambda_{t}
$$

then Girsanov's theorem implies that under $P, v_{t}$ is a standard $n$-dimensional Brownian motion if we define

$$
d v_{t}=D_{t}^{-1}\left(d y_{t}-C_{t} x_{t} d t\right), \quad v_{0}=0 .
$$

That is, under $P$,

$$
d y_{t}=C_{t} x_{t} d t+D_{t} d v_{t}
$$

Note that under $P$, the process $\left\{x_{t}\right\}$ still satisfies (2.1). Consequently, under $P$ the processes $\left\{x_{t}\right\}$ and $\left\{y_{t}\right\}$ satisfy the real world dynamics (2.1) and (2.2). However, $\bar{P}$ is a more convenient measure with which to work.

Below, we assume that $g: \mathbb{R}^{m} \rightarrow \mathbb{R}$ is an arbitrary "test" function, which is in $\mathbb{C}^{2}$ and has compact support.

Filtering is concerned with estimates of the form $\mathbf{E}\left\{g\left(x_{t}\right) \mid \mathcal{Y}_{t}\right\}$. Using a version of Bayes's theorem [6], we have

$$
\mathbf{E}\left\{g\left(x_{t}\right) \mid \mathcal{Y}_{t}\right\}=\frac{\overline{\mathbf{E}}\left\{\Lambda_{t} g\left(x_{t}\right) \mid \mathcal{Y}_{t}\right\}}{\overline{\mathbf{E}}\left\{\Lambda_{t} \mid \mathcal{Y}_{t}\right\}}
$$

Write $\sigma(g)_{t}=\overline{\mathbf{E}}\left\{\Lambda_{t} g\left(x_{t}\right) \mid \mathcal{Y}_{t}\right\}$ so that

$$
\mathbf{E}\left\{g\left(x_{t}\right) \mid \mathcal{Y}_{t}\right\}=\frac{\sigma(g)_{t}}{\sigma(1)_{t}} .
$$

Consequently, $\sigma(g)_{t}$ is a measure-valued process; it is an unnormalized conditional expectation of $g\left(x_{t}\right)$ given $\mathcal{Y}_{t}$. 
Suppose that $\sigma(\cdot)_{t}$ has a density $\alpha_{t}(x)$; then

$$
\sigma(g)_{t}=\overline{\mathbf{E}}\left\{\Lambda_{t} g\left(x_{t}\right) \mid \mathcal{Y}_{t}\right\}=\int_{\mathbb{R}^{m}} g(x) \alpha_{t}(x) d x .
$$

We now give a proof of the well-known Zakai equation for $\alpha$.

THEOREM 2.1.

$$
\begin{aligned}
\alpha_{t}(x)= & \alpha_{0}(x)-\int_{0}^{t} \operatorname{div}\left(A_{s} x \alpha_{s}(x)\right) d s+\frac{1}{2} \int_{0}^{t} \operatorname{Tr}\left(\nabla^{2} \alpha_{s}(x) B_{s} B_{s}^{0}\right) d s \\
& +\int_{0}^{t} \alpha_{s}(x) x^{0} C_{s}^{0}\left(D_{s}^{-1}\right)^{0} D_{s}^{-1} d y_{s},
\end{aligned}
$$

where $\alpha_{0}(x)=\pi_{o}(x)$, the density of $x_{0}$.

Proof. From Ito's rule

$$
\begin{aligned}
g\left(x_{t}\right)= & g\left(x_{0}\right)+\int_{0}^{t}\left(\nabla g\left(x_{s}\right)\right)^{0} A_{s} x_{s} d s+\int_{0}^{t}\left(\nabla g\left(x_{s}\right)\right)^{0} B_{s} d w_{s} \\
& +\frac{1}{2} \int_{0}^{t} \operatorname{Tr}\left(\nabla^{2} g\left(x_{s}\right) B_{s} B_{s}^{0}\right) d s .
\end{aligned}
$$

From (2.5) and (2.8)

$$
\begin{aligned}
\Lambda_{t} g\left(x_{t}\right)= & g\left(x_{0}\right)+\int_{0}^{t} \Lambda_{s}\left(\nabla g\left(x_{s}\right)\right)^{0} A_{s} x_{s} d s+\int_{0}^{t} \Lambda_{s}\left(\nabla g\left(x_{s}\right)\right)^{0} B_{s} d w_{s} \\
& +\frac{1}{2} \int_{0}^{t} \Lambda_{s} \operatorname{Tr}\left(\nabla^{2} g\left(x_{s}\right) B_{s} B_{s}^{0}\right) d s+\int_{0}^{t} \Lambda_{s} g\left(x_{s}\right) x_{s}^{0} C_{s}^{0}\left(D_{s}^{-1}\right)^{0} D_{s}^{-1} d y_{s}
\end{aligned}
$$

Conditioning each side of (2.9) on $\mathcal{Y}_{t}$ (see Lemma 3.2, p. 261, of [13]) we have

$$
\begin{aligned}
\sigma(g)_{t}= & \sigma(g)_{0}+\int_{0}^{t} \sigma\left((\nabla g)^{0} A x\right)_{s} d s+\frac{1}{2} \int_{0}^{t} \sigma\left(\operatorname{Tr}\left(\nabla^{2} g\right) B_{s} B_{s}^{0}\right) d s \\
& +\int_{0}^{t} \sigma\left(g x^{9}\right)_{s} C_{s}^{0}\left(D_{s}^{-1}\right)^{0} D_{s}^{-1} d y_{s} .
\end{aligned}
$$

Integrating each term by parts as on p. 277 of [7] gives (2.7).

Remarks. The Zakai equation (2.7) is a stochastic partial differential equation for the unnormalized conditional density of $x_{t}$ given $\mathcal{Y}_{t}$. In general, the solution of this equation is a conditional density function, evolving stochastically in time. For the linear, Gaussian dynamics (2.1) and (2.2), however, $\alpha_{t}(x)$ has a simple form. In fact (see [10] or [11]),

$$
\alpha_{t}(x)=\frac{\nu_{t}}{(2 \pi)^{m / 2}\left|\Sigma_{t}\right|^{1 / 2}} \exp \left(-\frac{1}{2}\left(x-m_{t}\right)^{0} \Sigma_{t}^{-1}\left(x-m_{t}\right)\right) .
$$

Here $m_{t}=\mathbf{E}\left\{x_{t} \mid \mathcal{Y}_{t}\right\}, m_{0}=\hat{x}_{0}, \Sigma_{t}=\mathbf{E}\left\{\left(x_{t}-m_{t}\right)\left(x_{t}-m_{t}\right) 9 \mathcal{Y}_{t}\right\}, \Sigma_{0}=P_{0}$, and $\nu_{t}$ is a normalizing factor.

It is well known that $m_{t}$ and $\Sigma_{t}$ are given by the Kalman filter equations

$$
\begin{aligned}
d m_{t} & =A_{t} m_{t} d t+\Sigma_{t} C_{t}^{0}\left(D_{t}^{-1}\right)^{0} D_{t}^{-1}\left(d y_{t}-C_{t} m_{t} d t\right), \\
\dot{\Sigma}_{t} & =\Sigma_{t} A_{t}^{0}+A_{t} \Sigma_{t}+B_{t} B_{t}^{0}-\Sigma_{t} C_{t}^{0}\left(D_{t}^{-1}\right)^{0} D_{t}^{-1} C_{t} \Sigma_{t} .
\end{aligned}
$$


Note that $\Sigma_{t}$ is deterministic and can be computed off-line. Also,

$$
\nu_{t}=(2 \pi)^{m / 2} \exp \left(\int_{0}^{t} m_{s}^{0} C_{s}^{0}\left(D_{s}^{-1}\right)^{0} D_{s}^{-1} d y_{s}-\frac{1}{2} \int_{0}^{t} m_{s}^{0} C_{s}^{0}\left(D_{s}^{-1}\right)^{0} D_{s}^{-1} C_{s} m_{s} d s\right) .
$$

3. Finite-dimensional filters. Let $e_{i}, e_{j} \in \mathbb{R}^{m}$ denote unit vectors with 1 in the $i$ th and $j$ th positions, respectively. Write

$$
\begin{aligned}
H_{t}^{i j} & =\int_{0}^{t}\left\langle x_{s}, e_{i}\right\rangle\left\langle e_{j}, x_{s}\right\rangle d s=\int_{0}^{t} x_{s}^{0}\left(e_{i} e_{j}^{0}\right) x_{s} d s, \\
L_{t}^{i j} & =\int_{0}^{t}\left\langle x_{s}, e_{i}\right\rangle\left\langle e_{j}, d x_{s}\right\rangle=\int_{0}^{t} x_{s}^{0}\left(e_{i} e_{j}^{0}\right) d x_{s}
\end{aligned}
$$

here $\langle\cdot, \cdot\rangle$ denotes the scalar product.

Also let $f_{j} \in \mathbb{R}^{n}$ denote the unit vector with 1 in the $j$ th position. Write

$$
J_{t}^{i j}=\int_{0}^{t}\left\langle x_{s}, e_{i}\right\rangle\left\langle f_{j}, d y_{s}\right\rangle=\int_{0}^{t} x_{s}^{0}\left(e_{i} f_{j}^{0}\right) d y_{s} .
$$

The parameters of our model are the entries in the matrices $A_{t}, B_{t}, C_{t}$, and $D_{t}$. To estimate these parameters using the EM algorithm (see section 5), it is necessary to obtain filtered estimates of these processes. That is, we wish to obtain expressions for

$$
\mathbf{E}\left\{H_{t}^{i j} \mid \mathcal{Y}_{t}\right\}, \quad \mathbf{E}\left\{L_{t}^{i j} \mid \mathcal{Y}_{t}\right\}, \quad \mathbf{E}\left\{J_{t}^{i j} \mid \mathcal{Y}_{t}\right\}
$$

Previously, these estimates have been obtained by smoothing procedures. For example,

$$
\mathbf{E}\left\{H_{t}^{i j} \mid \mathcal{Y}_{t}\right\}=\int_{0}^{t} \mathbf{E}\left\{x_{s}^{0}\left(e_{i} e_{j}\right) x_{s} \mid \mathcal{Y}_{t}\right\} d s
$$

However, this involves a considerable memory requirement. In this section we prove the remarkable result that the filtered estimates for $H_{t}^{i j}, L_{t}^{i j}$, and $J_{t}^{i j}$ can be described in terms of a finite number of statistics.

Motivated by the techniques in [6], we define a measure associated with $H_{t}^{i j}$ as follows.

DEFINITION 3.1. For any test function $g: \mathbb{R}^{m} \rightarrow \mathbb{R}$, define a measure-valued process $\overline{\mathbf{E}}\left\{\Lambda_{t} H_{t}^{i j} g\left(x_{t}\right) \mid \mathcal{Y}_{t}\right\}$. This has a density $\beta_{t}^{i j}(x)$ so that

$$
\overline{\mathbf{E}}\left\{\Lambda_{t} H_{t}^{i j} g\left(x_{t}\right) \mid \mathcal{Y}_{t}\right\}=\int_{\mathbb{R}^{m}} \beta_{t}^{i j}(x) g(x) d x .
$$

The existence of the density $\beta_{t}^{i j}(x)$ follows from the existence and uniqueness of solutions of stochastic partial differential equations such as (3.9). This is established in section 4.2 of [10] and on page 140 of [12].

The following theorem shows the surprising result that we can describe the measure $\beta_{t}^{i j}(x)$ exactly as a quadratic in $x$ multiplying the $\alpha_{t}(x)$ of $(2.7)$.

THEOREM 3.2. At time $t$, the density $\beta_{t}^{i j}(x)$ is completely described by the five statistics $a_{t}^{i j}, b_{t}^{i j}, c_{t}^{i j}, \Sigma_{t}$, and $m_{t}$ as follows:

$$
\beta_{t}^{i j}(x)=\left(a_{t}^{i j}+x^{0} b_{t}^{i j}+x^{0} c_{t}^{i j} x\right) \alpha_{t}(x) .
$$


Here $a_{t}^{i j} \in \mathbb{R}, b_{t}^{i j} \in \mathbb{R}^{m}$, and $c_{t}^{i j} \in L_{s}\left(\mathbb{R}^{m}, \mathbb{R}\right)$, the space of symmetric $m \times m$ matrices. Further,

$$
\begin{aligned}
\frac{d a_{t}^{i j}}{d t}=\operatorname{Tr}\left(c_{t}^{i j} B_{t} B_{t}^{0}\right)+b_{t}^{i j 0} B_{t} B_{t}^{0} \Sigma_{t}^{-1} m_{t}, \quad a_{0}^{i j}=0 \in \mathbb{R}, \\
\frac{d b_{t}^{i j}}{d t}=-\left(A_{t}^{0}+\Sigma_{t}^{-1} B_{t} B_{t}^{0}\right) b_{t}^{i j}+2 c_{t}^{i j} B_{t} B_{t}^{0} \Sigma_{t}^{-1} m_{t}, \quad b_{0}^{i j}=0 \in \mathbb{R}^{m}, \\
\frac{d c_{t}^{i j}}{d t}=-\left(A_{t}^{0}+\Sigma_{t}^{-1} B_{t} B_{t}^{0}\right) c_{t}^{i j}-c_{t}^{i j}\left(A_{t}+B_{t} B_{t}^{0} \Sigma_{t}^{-1}\right)+\frac{1}{2}\left(e_{j} e_{i}^{0}+e_{i} e_{j}^{0}\right), \\
c_{0}^{i j}=0 \in L_{s}\left(\mathbb{R}^{m}, \mathbb{R}\right) .
\end{aligned}
$$

Proof. First note that for any test function $g$, applying Ito's rule to (2.1) and $(2.5)$,

$$
\begin{aligned}
\Lambda_{t} H_{t}^{i j} g\left(x_{t}\right)= & \int_{0}^{t} \Lambda_{s} H_{s}^{i j}\left(\nabla g\left(x_{s}\right)\right)^{0} A_{s} x_{s} d s+\int_{0}^{t} \Lambda_{s} H_{s}^{i j}\left(\nabla g\left(x_{s}\right)\right)^{0} B_{s} d w_{s} \\
& +\frac{1}{2} \int_{0}^{t} \Lambda_{s} H_{s}^{i j} \operatorname{Tr}\left(\nabla^{2} g\left(x_{s}\right) B_{s} B_{s}^{0}\right) d s \\
& +\int_{0}^{t} \Lambda_{s} H_{s}^{i j} g\left(x_{s}\right) x_{s}^{0} C_{s}^{0}\left(D_{s}^{-1}\right)^{0} D_{s}^{-1} d y_{s} \\
& +\int_{0}^{t} \Lambda_{s} g\left(x_{s}\right) x_{s}^{0}\left(e_{i} e_{j}^{0}\right) x_{s} d s .
\end{aligned}
$$

Conditioning on $\mathcal{Y}_{t}$ under $\bar{P}$ (see Lemma 3.2, p. 261, of [13]) we have

$$
\begin{aligned}
\overline{\mathbf{E}}\left\{\Lambda_{t} H_{t}^{i j} g\left(x_{t}\right) \mid \mathcal{Y}_{t}\right\}= & \int_{0}^{t} \overline{\mathbf{E}}\left\{\Lambda_{s} H_{s}^{i j}\left(\nabla g\left(x_{s}\right)^{0} A_{s} x_{s}\right) \mid \mathcal{Y}_{s}\right\} d s \\
& +\frac{1}{2} \int_{0}^{t} \overline{\mathbf{E}}\left\{\Lambda_{s} H_{s}^{i j} \operatorname{Tr}\left(\nabla^{2} g\left(x_{s}\right) B_{s} B_{s}^{0}\right) \mid \mathcal{Y}_{s}\right\} d s \\
& +\int_{0}^{t} \overline{\mathbf{E}}\left\{\Lambda_{s} H_{s}^{i j} g\left(x_{s}\right) x_{s}^{0} C_{s}^{0}\left(D_{s}^{-1}\right)^{0} D_{s}^{-1} \mid \mathcal{Y}_{s}\right\} d y_{s} \\
& +\int_{0}^{t} \overline{\mathbf{E}}\left\{\Lambda_{s} g\left(x_{s}\right) x_{s}^{0}\left(e_{i} e_{j}^{0}\right) x_{s} \mid \mathcal{Y}_{s}\right\} d s .
\end{aligned}
$$

That is, in terms of the densities $\beta_{t}^{i j}(x)$ and $\alpha_{t}(x)$,

$$
\begin{aligned}
\int_{\mathbb{R}^{m}} \beta_{t}^{i j}(x) g(x) d x= & \int_{0}^{t} \int_{\mathbb{R}^{m}} \beta_{s}^{i j}(x)(\nabla g(x))^{0} A_{s} x d x d s \\
& +\frac{1}{2} \int_{0}^{t} \int_{\mathbb{R}^{m}} \beta_{s}^{i j}(x) \operatorname{Tr}\left(\nabla^{2} g(x) B_{s} B_{s}^{0}\right) d x d s \\
& +\int_{0}^{t} \int_{\mathbb{R}^{m}} \beta_{s}^{i j}(x) g(x) x^{0} C_{s}^{0}\left(D_{s}^{-1}\right)^{0} D_{s}^{-1} d x d y_{s} \\
& +\int_{0}^{t} \int_{\mathbb{R}^{m}} \alpha_{s}(x) g(x) x^{0}\left(e_{i} e_{j}^{0}\right) x d x d s .
\end{aligned}
$$

Integrating by parts in $x$, because this equation holds for all test functions $g$, we see 
that $\beta_{t}^{i j}(x)$ must satisfy the stochastic partial differential equation:

$$
\begin{aligned}
\beta_{t}^{i j}(x)= & -\int_{0}^{t} \operatorname{div}\left(\beta_{s}^{i j}(x) A_{s} x\right) d s+\frac{1}{2} \int_{0}^{t} \operatorname{Tr}\left(\nabla^{2} \beta_{s}^{i j}(x) B_{s} B_{s}^{0}\right) d s \\
& +\int_{0}^{t} \beta_{s}^{i j}(x) x^{0} C_{s}^{0}\left(D_{s}^{-1}\right)^{0} D_{s}^{-1} d y_{s}+\int_{0}^{t} \alpha_{s}(x) x^{0}\left(e_{i} e_{j}^{0}\right) x d s .
\end{aligned}
$$

We look for a solution of (3.9) of the form

$$
\bar{\beta}_{s}(x)=\left(a_{s}^{i j}+x^{0} b_{s}^{i j}+x^{0} c_{s}^{i j} x\right) \alpha_{s}(x) .
$$

As noted just after Definition 3.1, if such a solution exists, it is unique.

To simplify notation we drop the superscripts $i, j$ on $a, b$, and $c$. Then

$$
\begin{aligned}
\operatorname{div}\left(\bar{\beta}_{s}(x) A_{s} x\right)= & \operatorname{div}\left(\left(a_{s}+b_{s}^{0} x+x^{0} c_{s} x\right) \alpha_{s}(x) A_{s} x\right) \\
= & \left(b_{s}+2 c_{s} x\right)^{0} A_{s} x \alpha_{s}(x)+\left(a_{s}+b_{s}^{0} x+x^{0} c_{s} x\right) \operatorname{div}\left(\alpha_{s}(x) A_{s} x\right), \\
\nabla \bar{\beta}_{s}(x)= & \nabla\left(\left(a_{s}+b_{s}^{0} x+x^{0} c_{s} x\right) \alpha_{s}(x)\right) \\
= & \left(b_{s}+2 c_{s} x\right) \alpha_{s}(x)+\left(a_{s}+b_{s}^{0} x+x^{0} c_{s} x\right) \nabla \alpha_{s}(x), \\
\nabla^{2} \bar{\beta}_{s}(x)= & 2 c_{s} \alpha_{s}(x)+2\left(b_{s}+2 c_{s} x\right)\left(\nabla \alpha_{s}(x)\right)^{0} \\
& +\left(a_{s}+b_{s}^{0} x+x^{0} c_{s} x\right) \nabla^{2} \alpha_{s}(x),
\end{aligned}
$$

$\operatorname{Tr}\left(\nabla^{2} \bar{\beta}_{s}(x) B_{s} B_{s}^{0}\right)=2 \alpha_{s}(x) \operatorname{Tr}\left(c_{s} B_{s} B_{s}^{0}\right)+2\left(b_{s}+2 c_{s} x\right)^{0} B_{s} B_{s}^{0} \nabla \alpha_{s}(x)$

$$
+\left(a_{s}+b_{s}^{0} x+x^{0} c_{s} x\right) \operatorname{Tr}\left(\nabla^{2} \alpha_{s}(x) B_{s} B_{s}^{0}\right) \text {. }
$$

Now from (2.11)

$$
\nabla \alpha_{s}(x)=-\Sigma_{s}^{-1}\left(x-m_{s}\right) \alpha_{s}(x) .
$$

Consequently, if we substitute $\bar{\beta}_{t}(x)$, given by (3.10) in the differential form of the right-hand side of (3.9), we obtain

$$
\begin{aligned}
-\left(b_{s}\right. & \left.+2 c_{s} x\right)^{0} A_{s} x \alpha_{s}(x) d s-\left(a_{s}+b_{s}^{0} x+x^{0} c_{s} x\right) \operatorname{div}\left(\alpha_{s}(x) A_{s} x\right) d s \\
& +\alpha_{s}(x) \operatorname{Tr}\left(c_{s} B_{s} B_{s}^{0}\right) d s-\left(b_{s}+2 c_{s} x\right)^{0} B_{s} B_{s}^{0} \Sigma_{s}^{-1}\left(x-m_{s}\right) \alpha_{s}(x) d s \\
& +\frac{1}{2}\left(a_{s}+b_{s}^{0} x+x^{0} c_{s} x\right) \operatorname{Tr}\left(\nabla^{2} \alpha_{s}(x) B_{s}^{0} B_{s}\right) d s \\
& +\left(a_{s}+b_{s}^{0} x+x^{0} c_{s} x\right) \alpha_{s}(x) x^{0} C_{s}^{0}\left(D_{s}^{-1}\right)^{0} D_{s}^{-1} d y_{s} \\
& +\alpha_{s}(x) x^{0}\left(e_{i} e_{j}^{0}\right) x d s .
\end{aligned}
$$

Also,

$$
d \bar{\beta}_{s}(x)=\left(d a_{s}+d b_{s}^{0} x+x^{0} d c_{s} x\right) \alpha_{s}(x)+\left(a_{s}+b_{s}^{0} x+x^{0} c_{s} x\right) d \alpha_{s}(x) .
$$

Consequently, $\bar{\beta}_{s}(x)$, given by (3.10), is a solution of (3.9) if (3.11) equals (3.12). However, $\alpha_{s}(x)$ solves the Zakai equation (2.7), so

$d \alpha_{s}(x)=-\operatorname{div}\left(\alpha_{s}(x) A_{s} x\right) d s+\frac{1}{2} \operatorname{Tr}\left(\nabla^{2} \alpha_{s}(x) B_{s} B_{s}^{0}\right) d s+\alpha_{s}(x) x^{0} C_{s}^{0}\left(D_{s}^{-1}\right)^{0} D_{s}^{-1} d y_{s}$.

Therefore, substituting the above expression for $d \alpha_{s}(x)$ into (3.12) yields

$$
\begin{aligned}
d \bar{\beta}_{s}(x)= & \left(d a_{s}+d b_{s}^{0} x+x^{0} d c_{s} x\right) \alpha_{s}(x) \\
& -\left(a_{s}+b_{s}^{0} x+x^{0} c_{s} x\right) \operatorname{div}\left(\alpha_{s}(x) A_{s} x\right) d s \\
& +\frac{1}{2}\left(a_{s}+b_{s}^{0}+x^{0} c_{s} x\right) \operatorname{Tr}\left(\nabla^{2} \alpha_{s}(x) B_{s} B_{s}^{0}\right) d s \\
& +\left(a_{s}+b_{s}^{0} x+x^{0} c_{s} x\right) x^{0} C_{s}^{0}\left(D_{s}^{-1}\right)^{0} D_{s}^{-1} \alpha_{s}(x) d y_{s} .
\end{aligned}
$$


Finally equating the coefficients of $x, x^{0}, x$ and the constants in (3.11) and (3.13), we see that the result holds if (3.5), (3.6), and (3.7) hold.

We now explicitly solve the ordinary differential equations (3.6) and (3.7).

Write $G_{t}$ for the matrix solution of

$$
\frac{d G_{t}}{d t}=-\left(A_{t}^{0}+\Sigma_{t}^{-1} B_{t} B_{t}^{0}\right) G_{t}, \quad G_{0}=I_{m \times m} .
$$

Note that $G_{t}$ is deterministic and can be calculated off-line. Also, as an exponential matrix, $G_{t}$ has an inverse $G_{t}^{-1}$.

LEMmA 3.3. The explicit solutions of (3.6) and (3.7) are

$$
\begin{aligned}
b_{t}^{i j} & =2 G_{t}\left(\int_{0}^{t} G_{s}^{-1} c_{s}^{i j} B_{s} B_{s}^{0} \Sigma_{s}^{-1} m_{s} d s\right), \\
c_{t}^{i j} & =\frac{1}{2} G_{t}\left(\int_{0}^{t} G_{s}^{-1}\left(e_{j} e_{i}^{0}+e_{i} e_{j}^{0}\right)\left(G_{s}^{0}\right)^{-1} d s\right) G_{t}^{0} .
\end{aligned}
$$

Proof. The above equations follow using variation of constants.

Remark. We proceed similarly with the process $J_{t}^{i j}$ and $L_{t}^{i j}$, omitting details.

DeFINITION 3.4. For any test function $g \in \mathbb{C}_{0}^{2}\left(\mathbb{R}^{m}\right)$ define the measure-valued process $\overline{\mathbf{E}}\left\{\Lambda_{t} J_{t}^{i j} g\left(x_{t}\right) \mid \mathcal{Y}_{t}\right\}$. From the results of [10] and [12], this has a density $\gamma_{t}^{i j}(x)$ so that

$$
\overline{\mathbf{E}}\left\{\Lambda_{t} J_{t}^{i j} g\left(x_{t}\right) \mid \mathcal{Y}_{t}\right\}=\int_{\mathbb{R}^{m}} \gamma_{t}^{i j}(x) g(x) d x .
$$

THEOREM 3.5. At time $t$, the density $\gamma_{t}^{i j}(x)$ is completely described by the five statistics $\bar{a}_{t}^{i j}, \bar{b}_{t}^{i j} \bar{c}_{t}^{i j}, \Sigma_{t}$, and $m_{t}$ as follows:

$$
\gamma_{t}^{i j}(x)=\left(\bar{a}_{t}^{i j}+x^{0} \bar{b}_{t}^{i j}+x^{0} \bar{c}_{t}^{i j} x\right) \alpha_{t}(x) .
$$

Here, $\bar{a}_{t}^{i j} \in \mathbb{R}, \bar{b}_{t}^{i j} \in \mathbb{R}^{m}$, and $\bar{c}_{t}^{i j} \in L_{s}\left(\mathbb{R}^{m}, \mathbb{R}^{m}\right)$. Further,

$$
\frac{d \bar{a}_{t}^{i j}}{d t}=\operatorname{Tr}\left(\bar{c}_{t}^{i j} B_{t} B_{t}^{0}\right)+\bar{b}_{t}^{i j 0} B_{t} B_{t}^{0} \Sigma_{t}^{-1} m_{t}, \quad \bar{a}_{0}^{i j}=0 \in \mathbb{R},
$$

$$
d \bar{b}_{t}^{i j}=\left[-\left(A_{t}^{0}+\Sigma_{t}^{-1} B_{t} B_{t}^{0}\right) \bar{b}_{t}^{i j}+2 \bar{c}_{t}^{i j} B_{t} B_{t}^{0} \Sigma_{t}^{-1} m_{t}\right] d t+d y_{t}^{0} f_{j} e_{i}, \quad b_{0}^{i j}=0 \in \mathbb{R}^{m},
$$

$$
\begin{aligned}
& \frac{d \bar{c}_{t}^{i j}}{d t}=-\left(A_{t}^{0}+\Sigma_{t}^{-1} B_{t} B_{t}^{0}\right) \bar{c}_{t}^{i j}-\bar{c}_{t}^{i j}\left(A_{t}+B_{t} B_{t}^{0} \Sigma_{t}^{-1}\right)+\frac{1}{2}\left(e_{i} f_{j}^{0} C_{t}+C_{t}^{0} f_{j} e_{i}^{0}\right), \\
& \quad \bar{c}_{0}^{i j}=0 \in L_{s}\left(\mathbb{R}^{m}, \mathbb{R}^{m}\right) .
\end{aligned}
$$

Proof. The product $\Lambda_{t} J_{t}^{i j} g\left(x_{t}\right)$ is calculated and each side conditioned on $\mathcal{Y}_{t}$. After integration by parts, the following stochastic partial differential equation is obtained for $\gamma_{t}^{i j}(x)$ :

$$
\begin{aligned}
d \gamma_{t}^{i j}(x)= & -\operatorname{div}\left(\gamma_{t}^{i j}(x) A_{t} x\right) d t+\frac{1}{2} \operatorname{Tr}\left(\nabla^{2} \gamma_{t}^{i j}(x) B_{t} B_{t}^{0}\right) d t+\gamma_{t}^{i j} x^{0} C_{t}^{0}\left(D_{t}^{0}\right)^{-1} D_{t}^{-1} d y_{t} \\
& +\alpha_{t}(x) x^{0} e_{i} f_{j}^{0} d y_{t}+\alpha_{t}(x)\left(x^{0} C_{t}^{0} f_{j} e_{i}^{0} x\right) d t
\end{aligned}
$$


Recalling that $\alpha_{t}(x)$ satisfies the Zakai equation (2.7), we see that

$$
\left(\bar{a}_{t}^{i j}+x^{0} \bar{b}_{t}^{i j} x+x^{0} \bar{c}_{t}^{i j} x\right) \alpha_{t}(x)
$$

is a solution of (3.21) if $\bar{a}_{t}^{i j}, \bar{b}_{t}^{i j}$, and $\bar{c}_{t}^{i j}$ satisfy (3.18), (3.19), and (3.20), respectively.

We now obtain explicit solutions to the above equations. Note that $f_{j}^{0} d y_{t}=$ $d y_{t}^{0} f_{j}=d y_{t}^{j}$, where $y_{t}^{j}$ denotes the $j$ th component of $y_{t}$.

LEMMA 3.6. The explicit solutions of (3.19) and (3.20) are

$$
\bar{b}_{t}^{i j}=2 G_{t}\left(\int_{0}^{t} G_{s}^{-1} \bar{c}_{s}^{i j} B_{s} B_{s}^{0} \Sigma_{s}^{-1} m_{s} d s+\int_{0}^{t} G_{s}^{-1} e_{i} d y_{s}^{j}\right)
$$

and

$$
\bar{c}_{t}^{i j}=\frac{1}{2} G_{t}\left(\int_{0}^{t} G_{s}^{-1}\left(e_{i} f_{j}^{0} C_{s}+C_{s}^{0} f_{j} e_{i}^{0}\right)\left(G_{s}^{-1}\right)^{0} d s\right) G_{t}^{0} .
$$

DeFinition 3.7. For any test function $g \in \mathbb{C}_{0}^{2}\left(\mathbb{R}^{m}\right)$ define the measure-valued process $\overline{\mathbf{E}}\left\{\Lambda_{t} L_{t}^{i j} g\left(x_{t}\right) \mid \mathcal{Y}_{t}\right\}$. From the results of [10] and [12], this has a density $\lambda_{t}^{i j}(x)$ so that

$$
\overline{\mathbf{E}}\left\{\Lambda_{t} L_{t}^{i j} g\left(x_{t}\right) \mid \mathcal{Y}_{t}\right\}=\int_{\mathbb{R}^{m}} \lambda_{t}^{i j}(x) g(x) d x .
$$

THEOREM 3.8. At time $t$, the density $\lambda_{t}^{i j}(x)$ is completely characterized by the five statistics $r_{t}^{i j}, s_{t}^{i j}$, and $u_{t}^{i j}$ as follows:

$$
\lambda_{t}^{i j}(x)=\left(r_{t}^{i j}+x^{0} s_{t}^{i j} x+x^{0} u_{t}^{i j} x\right) \alpha_{t}(x) .
$$

Here, $r_{t}^{i j} \in \mathbb{R}, s_{t}^{i j} \in \mathbb{R}^{m}$, and $u_{t}^{i j} \in L_{s}\left(\mathbb{R}^{n}, \mathbb{R}^{m}\right)$. Further,

$$
\begin{gathered}
\frac{d r_{t}^{i j}}{d t}=\operatorname{Tr}\left(u_{t}^{i j} B_{t} B_{t}^{0}\right)+s_{t}^{i j 0} B_{t} B_{t}^{0} \Sigma_{t}^{-1} m_{t}-\operatorname{Tr}\left(B_{t} B_{t}^{0} e_{i} e_{j}^{0}\right), \quad r_{0}^{i j}=0 \in \mathbb{R}, \\
\frac{d s_{t}^{i j}}{d t}=-\left(A_{t}^{0}+\Sigma_{t}^{-1} B_{t} B_{t}^{0} s_{t}^{i j}+2 u_{t}^{i j} B_{t} B_{t}^{0} \Sigma_{t}^{-1} m_{t}\right. \\
-\left(e_{j} e_{i}^{0} B_{t} B_{t}^{0} \Sigma_{t}^{-1} m_{t}, \quad s_{0}^{i j}=0 \in \mathbb{R}^{m}\right. \\
\frac{d u^{i j}}{d t}=-\left(A_{t}^{0}+\Sigma_{t}^{-1} B_{t} B_{t}^{0}\right) u_{t}^{i j}-u_{t}^{i j}\left(A_{t}+B_{t} B_{t}^{0} \Sigma_{t}^{-1}\right) \\
+\frac{1}{2}\left(e_{i} e_{j}^{0}\left(A_{t}+B_{t} B_{t}^{0} \Sigma_{t}^{-1}\right)+\left(A_{t}^{0}+\Sigma_{t}^{-1} B_{t} B_{t}^{0}\right) e_{j} e_{i}^{0}\right) \\
u_{0}^{i j}=0 \in L_{s}\left(\mathbb{R}^{m}, \mathbb{R}^{m}\right) .
\end{gathered}
$$

Proof. The product $\Lambda_{t} L_{t}^{i j} g\left(x_{t}\right)$ is calculated and each side conditioned on $\mathcal{Y}_{t}$. After integration by parts, the following stochastic partial differential equation is obtained for $\lambda_{t}^{i j}(x)$ :

$$
\begin{aligned}
d \lambda_{t}^{i j}(x)= & -\operatorname{div}\left(\lambda_{t}^{i j}(x) A_{t} x\right) d t \\
& +\frac{1}{2} \operatorname{Tr}\left(\nabla^{2} \lambda_{t}^{i j}(x) B_{t} B_{t}^{0}\right) d t+\lambda_{t}^{i j}(x) x^{0} C_{t}^{0}\left(D_{t}^{-1}\right)^{0} D_{t}^{-1} d y_{t} \\
& +\alpha_{t}(x) x^{0} e_{i} e_{j}^{0} A_{t} x d t-\operatorname{div}\left(x^{0} e_{i} e_{j}^{0} B_{t} B_{t}^{0} \alpha_{t}(x)\right) d t .
\end{aligned}
$$


We see that

$$
\left(r_{t}^{i j}+x^{0} s_{t}^{i j} x+x^{0} u_{t}^{i j} x\right) \alpha_{t}(x)
$$

solves (3.28) if $r_{t}^{i j}, s_{t}^{i j}$, and $u_{t}^{i j}$ satisfy (3.25), (3.26), and (3.27) .

Again, (3.26) and (3.27) can be solved by variation of constants. We summarize this in the following lemma.

LEMMA 3.9. The explicit solutions of (3.26) and (3.27) are

$$
\begin{aligned}
s_{t}^{i j}=G_{t}\left(\int_{0}^{t} G_{s}^{-1}\left(2 u_{s}^{i j}-e_{j} e_{i}^{0}\right) \Sigma_{s}^{-1} B_{s} B_{s}^{0} m_{s} d s\right) \\
u_{t}^{i j}=\frac{1}{2} G_{t}\left(\int _ { 0 } ^ { t } \left[G _ { s } ^ { - 1 } \left(e_{i} e_{j}^{0}\left(A_{s}+B_{s} B_{s}^{0} \Sigma_{s}^{-1}\right)\right.\right.\right. \\
\left.\left.+\left(A_{s}^{0}+\Sigma_{s}^{-1} B_{s} B_{s}^{0}\right) e_{j} e_{i}^{0}\left(G_{s}^{-1}\right)^{0}\right] d s\right) G_{t} .
\end{aligned}
$$

Remark. We observe from the definition of $G_{t},(3.14)$, that the integrand in (3.30) includes only half of the four terms in the derivative of $G_{t}^{-1}\left(e_{i} e_{j}^{0}+e_{j} e_{i}^{0}\right)\left(G_{t}^{-1}\right)^{0}$, and so the integral cannot be evaluated in closed form.

THEOREM 3.10. Finite-dimensional filters for $H_{t}^{i j}, J_{t}^{i j}$, and $L_{t}^{i j}$ defined in (3.1), (3.3), and (3.2) are given by

$$
\begin{aligned}
& \mathbf{E}\left\{H_{t}^{i j} \mid \mathcal{Y}_{t}\right\}=a_{t}^{i j}+m_{t}^{0} b_{t}^{i j}+\sum_{p=1}^{m} \sum_{q=1}^{m} c_{t}^{i j}(p, q) \Sigma_{t}(p, q)+m_{t}^{0} c_{t}^{i j} m_{t}, \\
& \mathbf{E}\left\{J_{t}^{i j} \mid \mathcal{Y}_{t}\right\}=\bar{a}_{t}^{i j}+m_{t}^{0} b_{t}^{i j}+\sum_{p=1}^{m} \sum_{q=1}^{m} \bar{c}_{t}^{i j}(p, q) \Sigma_{t}(p, q)+m_{t}^{0} \bar{c}_{t}^{i j} m_{t}, \\
& \mathbf{E}\left\{L_{t}^{i j} \mid \mathcal{Y}_{t}\right\}=r_{t}^{i j}+m_{t}^{0} s_{t}^{i j}+\sum_{p=1}^{m} \sum_{q=1}^{m} u_{t}^{i j}(p, q) \Sigma_{t}(p, q)+m_{t}^{0} u_{t}^{i j} m_{t} .
\end{aligned}
$$

Proof. Recall from (2.11) that $\alpha_{t}$ is an unnormalized Gaussian density with mean $m_{t}$ and variance $\Sigma_{t}$. Therefore,

$$
\int_{\mathbb{R}^{m}} \alpha_{t}(x) d x=\nu_{t}
$$

Note that for any $k \in \mathbb{R}^{m}$

$$
\int_{\mathbb{R}^{m}} k^{0} x \alpha_{t}(x) d x=\left(k^{0} m_{t}\right) \nu_{t} .
$$

Also for any matrix $M \in L\left(\mathbb{R}^{m}, \mathbb{R}^{m}\right)$ with entries $M(p, q), 1 \leq p, q \leq m$,

$$
\begin{aligned}
\int_{\mathbb{R}^{m}} x^{0} M x \alpha_{t}(x) d x & =\int_{\mathbb{R}^{m}}\left(x-m_{t}\right)^{0} M\left(x-m_{t}\right) \alpha_{t}(x) d x+m_{t}^{0} M m_{t} \int_{\mathbb{R}^{m}} \alpha_{t}(x) d x \\
& =\left(\sum_{p=1}^{M} \sum_{q=1}^{M} M(p, q) \Sigma_{t}(p, q)+m_{t}^{0} M m_{t}\right) \nu_{t} .
\end{aligned}
$$


Now from Bayes's theorem (2.6), we have

$$
\begin{aligned}
\mathbf{E}\left\{H_{t}^{i j} \mid \mathcal{Y}_{t}\right\} & =\frac{\overline{\mathbf{E}}\left\{\Lambda_{t} H_{t}^{i j} \mid \mathcal{Y}_{t}\right\}}{\overline{\mathbf{E}}\left\{\Lambda_{t} \mid \mathcal{Y}_{t}\right\}} \\
& =\frac{\int_{\mathbb{R}^{m}} \beta_{t}^{i j}(x) d x}{\int_{\mathbb{R}^{m}} \alpha_{t}(x) d x} \\
& =a_{t}^{i j}+m_{t}^{0} b_{t}^{i j}+\sum_{p=1}^{m} \sum_{q=1}^{m} c_{t}^{i j}(p, q) \Sigma_{t}(p, q)+m_{t}^{0} c_{t}^{i j} m_{t}
\end{aligned}
$$

by (3.4) and because the factors $\nu_{t}$ cancel. The proofs of equations (3.32) and (3.33) are similar.

4. Finite-dimensional filter for higher order moments. The techniques of the previous section can be generalized to show that integrals and stochastic integrals of higher moments of the state variables have filtered estimates which can be expressed in terms of a finite number of statistics. The results also hold for other functions of the state and will be investigated in a subsequent paper.

Assumption 4.1. For notational simplicity, in this section we assume that the state and observation processes are scalar valued, i.e., that $m=n=1$ in (2.1) and (2.2).

Let $\Gamma_{t}$ be the process defined as

$$
\Gamma_{t}=\int_{0}^{t} x_{s}^{p} d s, \quad p \in \mathbb{Z}^{+}
$$

Suppose $g: \mathbb{R} \rightarrow \mathbb{R}$ is any test function and suppose for a density-valued process $\mu_{t}(x)$

$$
\overline{\mathbf{E}}\left\{\Lambda_{t} \Gamma_{t} g\left(x_{t}\right) \mid \mathcal{Y}_{t}\right\}=\int_{\mathbb{R}} \mu_{t}(x) g(x) d x .
$$

We now show that $\mathbf{E}\left\{\Gamma_{t} \mid \mathcal{Y}_{t}\right\}$ can be computed via a finite-dimensional filter.

TheOREM 4.2. At time $t$, the density $\mu_{t}$ is completely characterized by the $p+3$ statistics, $a_{t}(0), a_{t}(1), \ldots, a_{t}(p), \Sigma_{t}$, and $m_{t}$ as follows:

$$
\mu_{t}(x)=\left[\sum_{i=0}^{p} a_{t}(i) x^{p}\right] \alpha_{t}(x),
$$

where $a_{0}(i)=0, i=1, \ldots, p$, and

$$
\begin{aligned}
\frac{d a_{t}(p)}{d t}= & -p\left(A_{t}+\Sigma_{t}^{-1} B_{t}^{2}\right) a_{t}(p)+1, \\
\frac{d a_{t}(p-1)}{d t}= & -(p-1)\left(A_{t}+\Sigma_{t}^{-1} B_{t}^{2}\right) a_{t}(p-1)+p a_{t}(p) \Sigma_{t}^{-1} B_{t}^{2} m_{t}, \\
\frac{d a_{t}(i)}{d t}= & -i\left(A_{t}+\Sigma_{t}^{-1} B_{t}^{2}\right) a_{t}(i)+\frac{1}{2}(i+1)(i+2) a_{t}(i+2) \\
& +(i+1) a_{t}(i+1) \Sigma_{t}^{-1} B_{t}^{2}, \quad i=1, \ldots, p-2, \\
\frac{d a_{t}(0)}{d t}= & B_{t}^{2} a_{t}(2)+\Sigma_{t}^{-1} B_{t}^{2} a_{t}(1) m_{t} .
\end{aligned}
$$


Proof.

$$
\begin{aligned}
\Lambda_{t} \Gamma_{t} g\left(x_{t}\right)= & \int_{0}^{t} \Lambda_{s} \Gamma_{s} \nabla g\left(x_{s}\right) A_{s} x_{s} d s+\int_{0}^{t} \Lambda_{s} \Gamma_{s} \nabla g\left(x_{s}\right) B_{s} d w_{s} \\
& +\frac{1}{2} \int_{0}^{t} \Lambda_{s} \Gamma_{s} \nabla^{2} g\left(x_{s}\right) B_{s}^{2} d s \\
& +\int_{0}^{t} \Lambda_{s} \Gamma_{s} g\left(x_{s}\right) x_{s} C_{s} D_{s}^{-2} d y_{s}+\int_{0}^{t} \Lambda_{s} g\left(x_{s}\right) x_{s}^{p} d s
\end{aligned}
$$

Conditioning on $\mathcal{Y}_{t}$, as in [13],

$$
\begin{aligned}
\int_{\mathbb{R}} \mu_{t}(x) g(x) d x= & \int_{0}^{t} \int_{\mathbb{R}} \mu_{s}(x) \nabla g(x) A_{s} x d x d s+\frac{1}{2} \int_{0}^{t} \int_{\mathbb{R}} \mu_{s}(x) \nabla^{2} g(x) B_{s}^{2} d x d s \\
& +\int_{0}^{t} \int_{\mathbb{R}} \mu_{s}(x) g(x) x C_{s} D_{s}^{-2} d x d y_{s}+\int_{0}^{t} \int_{\mathbb{R}} x^{p} g(x) \alpha_{s}(x) d x d s .
\end{aligned}
$$

Integrating by parts in $x$, we see that $\mu_{t}(\cdot)$ satisfies the stochastic partial differential equation

$$
\begin{aligned}
\mu_{t}(x)= & -\int_{0}^{t} \frac{d}{d x}\left(\mu_{s}(x) A_{s} x\right) d s+\frac{1}{2} \int_{0}^{t} \frac{d^{2}}{d x^{2}} \mu_{s}(x) B_{s}^{2} d s+\int_{0}^{t} \mu_{s}(x) x C_{s} D_{s}^{-2} d y_{s} \\
& +\int_{0}^{t} x^{p} \alpha_{s}(x) d s .
\end{aligned}
$$

It can then be verified that (4.1) is a solution to the above equation if the time-varying coefficients $a_{t}(0), \ldots, a_{t}(p)$ satisfy the ordinary differential equations (4.2).

Remark. The ordinary differential equations (4.2) can be solved explicitly by variation of constants.

Finally, we note that a similar derivation to that given in this section yields finite-dimensional filters for $\int_{0}^{t} x_{s}^{p} d x_{s}$ and $\int_{0}^{t} x_{s}^{p} d y_{s}, p=1,2,3, \ldots$

5. Filtered EM algorithm for Gaussian state-space models. The aim of this section is to derive a filter-based EM algorithm for computing maximumlikelihood (ML) parameter estimates of a linear Gaussian state-space system. The finite-dimensional filters of section 3 are used in implementing the E-step of the EM algorithm, resulting in a filter-based EM algorithm.

Consider the time-invariant version of the state-space model $(2.1),(2.2)$ :

$$
\begin{aligned}
d x_{t} & =A x_{t} d t+B d w_{t} \\
d y_{t} & =C x_{t} d t+D d v_{t} .
\end{aligned}
$$

Our aim is to compute ML estimates of the parameters $\theta=(A, C)$ given the observations $\mathcal{Y}_{t}=\sigma\left\{y_{s}: s \leq t\right\}$ and assuming $B, D$ are known. We do this via the EM algorithm.

Remark. Unlike the discrete-time case, in continuous time, it is not possible to obtain ML estimates of the variance terms $B$ and $D$ because measures corresponding to Wiener processes with different variances are not absolutely continuous (see Chapter 6.1 in [13]). At the end of this section, we give estimates for $B$ and $D$ in terms of the quadratic variations of the state and observation processes. 
EM ALGORIThm. Suppose that we have observation history $\mathcal{Y}_{t}$ available. Let $\left\{P_{\theta}, \theta \in \Theta\right\}$ be a family of probability measures on $(\Omega, \mathcal{F})$, all absolutely continuous with respect to a fixed probability measure $P_{0}$. The log likelihood function for computing an estimate of the parameter $\theta$ based on the information available in $\mathcal{Y}_{t}$ is

$$
\mathcal{L}(\theta)=\mathbf{E}_{0}\left\{\log \frac{d P_{\theta}}{d P_{0}} \mid \mathcal{Y}_{t}\right\}
$$

and the maximum likelihood estimate (MLE) is defined by

$$
\widehat{\theta} \in \underset{\theta \in \Theta}{\operatorname{argmax}} \mathcal{L}(\theta) \text {. }
$$

The EM algorithm is an iterative numerical method for computing the MLE. Let $\hat{\theta}_{0}$ be the initial parameter estimate. The EM algorithm generates a sequence of parameter estimates as follows.

Each iteration of the EM algorithm consists of two steps:

Step 1 (E-step). Set $\tilde{\theta}=\widehat{\theta}_{j}$ and compute $Q(\cdot, \tilde{\theta})$, where

$$
Q(\theta, \tilde{\theta})=\mathbf{E}_{\tilde{\theta}}\left\{\log \frac{d P_{\theta}}{d P_{\tilde{\theta}}} \mid \mathcal{Y}_{t}\right\}
$$

Step 2 (M-step). Find $\widehat{\theta}_{j+1} \in \underset{\theta \in \Theta}{\operatorname{argmax}} Q\left(\theta, \theta_{j}\right)$.

The sequence-generated $\left\{\widehat{\theta}_{j}, j \geq 0\right\}$ gives nondecreasing values of $\mathcal{L}\left(\hat{\theta}_{j}\right)$ with equality if and only if $\widehat{\theta}_{j+1}=\widehat{\theta}_{j}$.

It is shown in the appendix that

$$
\begin{aligned}
Q(\theta, \tilde{\theta})= & \mathbf{E}\left\{\int _ { 0 } ^ { t } x _ { s } ^ { 0 } A ^ { 0 } \left[B B^{q^{\#}} d x_{s}-\frac{1}{2} \int_{0}^{t} x_{s}^{0} A^{0}\left[B B^{q^{\#}} A x_{s} d s \mid \mathcal{Y}_{t}\right\}\right.\right. \\
& +\mathbf{E}\left\{\int _ { 0 } ^ { t } x _ { s } ^ { 0 } C ^ { 0 } \left(D D^{9-1} d y_{s}-\frac{1}{2} \int_{0}^{t} x_{s}^{0} C^{0}\left(D D^{0-1} C x_{s} d s \mid \mathcal{Y}_{t}\right\}+\mathbf{E}\left\{R\left(\tilde{\theta} \mid \mathcal{Y}_{t}\right\},\right.\right.\right.
\end{aligned}
$$

where \# denotes the pseudoinverse and $R(\tilde{\theta})$ does not involve $\theta$.

To implement the M-step we set the derivatives $\partial Q / \partial \theta=0$. This yields

$$
\begin{aligned}
& A=\mathbf{E}\left\{\int_{0}^{t} d x_{s} x_{s}^{0} \mid \mathcal{Y}_{t}\right\}\left(\mathbf{E}\left\{\int_{0}^{t} x_{s} x_{s}^{0} d s \mid \mathcal{Y}_{t}\right\}\right)^{-1}=\hat{L}_{t}^{0} \hat{H}_{t}^{-1}, \\
& C=\mathbf{E}\left\{\int_{0}^{t} d y_{s} x_{s}^{0} \mid \mathcal{Y}_{t}\right\}\left(\mathbf{E}\left\{\int_{0}^{t} x_{s} x_{s}^{0} d s \mid \mathcal{Y}_{t}\right\}\right)^{-1}=\hat{J}_{t}^{0} \hat{H}_{t}^{-1}
\end{aligned}
$$

where $\hat{H}_{t}$ and $\hat{L}_{t} \in \mathbb{R}^{m \times m}$ denote matrices with elements $\hat{H}_{t}^{i j} \stackrel{4}{=} \mathbf{E}\left\{H_{t}^{i j} \mid \mathcal{Y}_{t}\right\}$ and $\hat{L}_{t}^{i j} \stackrel{4}{=} \mathbf{E}\left\{L_{t}^{i j} \mid \mathcal{Y}_{t}\right\}, i, j \in\{1, \ldots, m\}$. Also, $\hat{J}_{t} \in \mathbb{R}^{m \times n}$ denotes the matrix with elements $\hat{J}_{t}^{i j} \stackrel{4}{=} \mathbf{E}\left\{J_{t}^{i j} \mid \mathcal{Y}_{t}\right\}, i=1, \ldots, m, j=1, \ldots, n$. The terms $\hat{H}_{t}^{i j}, \hat{L}^{i j}$, and $\hat{J}_{t}^{i j}$ are computed using Theorems 3.2, 3.8, and 3.5 together with the filters in Theorem 3.10. Thus we have a filter-based EM algorithm.

Remark. We have presented the EM algorithm for updating the parameters of a general state-space model. However, we have not addressed identifiability issues of our state-space model. 
Indeed, identifiability and consistency of the ML estimator have been studied in special cases of our model (where our filter-based algorithm also applies). For example, in [9], $A$ is of a known structure but parametrized by an unknown vector $\theta$. Consistency of the ML estimators is proved in [16].

Estimation of $B$ and $D$. First consider the tensor product of $x_{t}$ with itself:

$$
x_{t} x_{t}^{0}=x_{0} x_{0}^{0}+\int_{0}^{t} x_{s} d x_{s}^{0}+\int_{0}^{t} d x_{s} x_{s}^{0}+\int_{0}^{t} B B^{0} d s .
$$

Conditioning both sides of (5.6) on $\mathcal{Y}_{t}$, we have

$$
\Sigma_{t}=\mathbf{E}\left\{x_{0} x_{0}^{0} \mid \mathcal{Y}_{t}\right\}+\mathbf{E}\left\{\int_{0}^{t} x_{s} d x_{s}^{0}+\int_{0}^{t} d x_{s} x_{s}^{0} \mid \mathcal{Y}_{t}\right\}+B B^{0} t
$$

$\mathbf{E}\left\{x_{0} x_{0}^{0} \mid \mathcal{Y}_{t}\right\}$ in (5.7) is the smoothed second moment and is given in terms of finitedimensional statistics; see Theorem 12.11, section 12.4 in [8]. The components of the conditioned stochastic integrals in (5.7) are given by the filtered estimates of $L_{t}^{i j}$. Consequently, we have a procedure for estimating the matrix $B B^{0}$.

Similarly, consider the tensor product of $y_{t}$ with itself:

$$
y_{t} y_{t}^{0}=\int_{0}^{t} y_{s} d y_{s}^{0}+\int_{0}^{t} d y_{s} y_{s}^{0}+D D^{0} t
$$

This expression simply amounts to evaluating $D D^{0}$ in terms of the quadratic variation of $y$.

6. Conclusion and extensions. For linear Gaussian dynamics, new finitedimensional filters have been derived which estimate integrals and stochastic integrals of the moments of the state variable. Used in the EM algorithm, these quantities provide maximum likelihood estimates of the parameters in the dynamics of the Kalman filter.

We mention two extensions of the results. First, similar filters can be derived for nonlinear systems with Benes-type nonlinearity. Details are presented in [21]. Second, techniques similar to those developed in this paper can be applied in the reconstruction of doubly stochastic autoregressive (AR) processes, as we now outline.

Assuming the scalar version $(m=n=1)$ of the state-space model (2.1), (2.2), consider the continuous-time doubly stochastic AR process $z_{t} \in \mathbb{R}$ defined as

$$
d z_{t}=f\left(x_{t}\right) z_{t} d t+d u_{t}, \quad z_{0}=1 .
$$

Here $u$ is a scalar Brownian process independent of $v$ and $w$, and $f: \mathbb{R} \rightarrow \mathbb{R}$ is a polynomial function. Suppose our aim is to derive a filter for computing $\mathbf{E}\left\{z_{t} \mid \mathcal{Y}_{t}\right\}$.

In (6.1), $z_{t}$ can be viewed as a continuous-time AR process with random coefficient $f\left(x_{t}\right)$, where $x_{t}$ itself evolves according to another AR process. In [4] we derived finite-dimensional filters for such problems in discrete time. The above model can be viewed as a continuous-time version of [4]. The problem of reconstructing $z_{t}$ when $x_{t}$ is a finite state Markov chain (instead of a continuous-valued Gaussian process) has recently been studied in the context of maneuvering target tracking [20]. Therefore, (6.1) can be viewed as the continuous-valued analogue of the model in [20].

Let us compute $\mathbf{E}\left\{z_{t} \mid \mathcal{Y}_{t}\right\}$. Note that we can solve (6.1) explicitly for $z_{t}$; this yields

$$
z_{t}=\exp \left(\int_{0}^{t} f\left(x_{s}\right) d s\right)\left[1+\int_{0}^{t} \exp \left(-\int_{0}^{s} f\left(x_{r}\right) d r\right) d u_{s}\right] .
$$


Since $u$ is independent of $x$ and $y$, our aim can be re-expressed as follows: compute the filtered estimate

$$
\mathbf{E}\left\{z_{t} \mid \mathcal{Y}_{t}\right\}=\mathbf{E}\left\{\exp \left(\int_{0}^{t} f\left(x_{s}\right) d s\right) \mid \mathcal{Y}_{t}\right\}
$$

Using techniques similar to those presented in this paper, it is possible to derive a finite-dimensional filter for $\mathbf{E}\left\{z_{t} \mid \mathcal{Y}_{t}\right\}$. Details are presented in [22].

Appendix A. Derivation of $\boldsymbol{Q}(\boldsymbol{\theta}, \tilde{\boldsymbol{\theta}})$. To update the estimate from $\tilde{A}$ to $A$, we employ Girsanov's theorem and introduce the density

$$
\begin{aligned}
\left.\frac{d P(A)}{d P(\tilde{A})}\right|_{\mathrm{G}_{t}}= & \exp \left(\int _ { 0 } ^ { t } x _ { s } ^ { 0 } \left(A^{0}-\tilde{A}^{9}\left(B B^{0}\right)^{\#} d x_{s}\right.\right. \\
& -\frac{1}{2} \int_{0}^{t} x_{s}^{0}\left(A^{0}-\tilde{A}^{9}\left(B B^{0}\right)^{\#}(A+\tilde{A}) x_{s} d s\right),
\end{aligned}
$$

where \# denotes the pseudoinverse. Then

$$
\begin{aligned}
\mathbf{E}\left\{\left.\log \frac{d P(A)}{d P(\tilde{A})}\right|_{\mathrm{G}_{t}} \mid \mathcal{Y}_{t}\right\}= & \mathbf{E}\left\{\int_{0}^{t} x_{s}^{0} A^{0}\left(B B^{\mathrm{q}}\right)^{\#} d x_{s}-\frac{1}{2} \int_{0}^{t} x_{s}^{0} A^{0}\left(B B^{0}\right)^{\#} A x_{s} d s \mid \mathcal{Y}_{t}\right\} \\
& +R(\tilde{A}),
\end{aligned}
$$

where $R(\tilde{A})$ does not involve $A$.

Similarly, to update the estimate from $\tilde{C}$ to $C$, we again apply Girsanov's theorem and introduce the density

$$
\begin{aligned}
\left.\frac{d P(C)}{d P(\tilde{C})}\right|_{\mathrm{G}_{t}}=\exp & \left(\int _ { 0 } ^ { t } x _ { s } ^ { 0 } \left(C^{0}-\tilde{C}^{9}\left(D D^{9-1} d y_{s}\right.\right.\right. \\
& -\frac{1}{2} \int_{0}^{t} x_{s}^{0}\left(C^{0}-\tilde{C}^{9}\left(D D^{9-1}(C+\tilde{C}) x_{s} d s\right) .\right.
\end{aligned}
$$

Consequently,

$$
\begin{aligned}
\mathbf{E}\left\{\left.\log \frac{d P(C)}{d P(\tilde{C})}\right|_{\mathrm{G}_{t}} \mid \mathcal{Y}_{t}\right\}= & \mathbf{E}\left\{\int _ { 0 } ^ { t } x _ { s } ^ { 0 } C ^ { 0 } \left(D D^{9^{-1}} d y_{s}\right.\right. \\
& -\frac{1}{2} \int_{0}^{t} x_{s}^{0} C^{0}\left(D D^{9^{-1}} C x_{s} d s \mid \mathcal{Y}_{t}\right\}+S(\tilde{C})
\end{aligned}
$$

where $S(\tilde{C})$ does not involve $C$.

Adding (A.1) and (A.2) yields (5.3).

\section{REFERENCES}

[1] R.H. Shumway And D.S. Stoffer, An approach to time series smoothing and forecasting using the EM algorithm, J. Time Series Anal., 3 (1982), pp. 253-264.

[2] D. GHosh, Maximum likelihood estimation of the dynamic shock-error model, J. Econometrics, 41 (1989), pp. 121-143.

[3] V. KRIShNAmuRthy, On-line estimation of dynamic shock-error models, IEEE Trans. Automat. Control, 35 (1994), pp. 1129-1134. 
[4] V. Krishnamurthy and R.J. Elliott, Exact finite dimensional filters for doubly stochastic auto-regressive processes, IEEE Trans. Automat. Control, June 1997 (to appear).

[5] R.J. ElliotT, Exact adaptive filters for Markov chains observed in Gaussian noise, Automatica, 30 (1994), pp. 1399-1408.

[6] R.J. Elliott, L. Aggoun, And J.B. Moore, Hidden Markov Models: Estimation and Control, Applications of Mathematics, Springer-Verlag, New York, 1995.

[7] R.J. ElliotT, Stochastic Calculus and Applications, Applications of Mathematics 18, SpringerVerlag, New York, 1982.

[8] R.S. Liptser And A.N. Shiryayev, Statistics of Random Processes 2, Springer-Verlag, Berlin, Heidelberg, New York, 1977.

[9] A. Dembo And O. Zeitouni, Parameter estimation of partially observed continuous-time stochastic processes via the EM algorithm, Stochastic Process. Appl., 23 (1986), pp. 91113.

[10] A. Bensoussan, Stochastic Control of Partially Observable Systems, Cambridge University Press, Cambridge, UK, 1992.

[11] A. Bensoussan and R.J. Elliott, General finite dimensional risk sensitive problems and small noise limits, IEEE Trans. Automat. Control, 41 (1996), pp. 210-215.

[12] E. PARdoux, Stochastic partial differential equations and filtering of diffusion processes, Stochastics, 3 (1979), pp. 127-167.

[13] E. Wong And B. HajeK, Stochastic Processes in Engineering Systems, Springer-Verlag, New York, 1985.

[14] A.P. Dempster, N.M. Laird, And D.B. Rubin, Maximum likelihood from incomplete data via the EM algorithm, J. Royal Statist. Soc. B, 39 (1977), pp. 1-38.

[15] C.F.J. Wu, On the convergence properties of the EM algorithm, Ann. Statist., 11 (1983), pp. 95-103.

[16] E.J. Hannan And M. Deistler, The Statistical Theory of Linear Systems, Wiley, New York, 1988.

[17] R.J. Elliott and V. Krishnamurthy, New finite dimensional filters for estimation of discrete-time linear Gaussian models, IEEE Trans. Automat. Control, submitted.

[18] D.L. Ocone, J.S. Baras, And S.I. Marcus, Explicit filters for diffusions with certain nonlinear drifts, Stochastics, 8 (1982), pp. 1-16.

[19] S.I. Marcus AND A.S. Willsky, Algebraic structure and finite dimensional nonlinear estimation, SIAM J. Math. Anal., 9 (1978), pp. 312-327.

[20] F. Dufour And P. Bertrand, An image based filter for discrete-time Markovian jump linear systems, Automatica, 32 (1996), pp. 241-247.

[21] R.J. Elliott, V. Krishamurthy, and H.V. Poor, Exact filters for certain moments and stochastic integrals of the state of systems with Benes nonlinearity, IEEE Trans. Automat. Control, submitted.

[22] V. KrishamuRthy AND R.J. Elliott, Exact finite dimensional filters for certain exponential functionals of Gaussian state-space processes, IEEE Trans. Automat. Control, submitted. 